\title{
Random walks on mutual microRNA-target gene interaction network improve the prediction of disease-associated microRNAs
}

\author{
Duc-Hau Le ${ }^{1}$, Lieven Verbeke ${ }^{2}$, Le Hoang Son ${ }^{3}$, Dinh-Toi Chu ${ }^{4,5}$ and Van-Huy Pham ${ }^{6 *}$
}

\begin{abstract}
Background: MicroRNAs (miRNAs) have been shown to play an important role in pathological initiation, progression and maintenance. Because identification in the laboratory of disease-related miRNAs is not straightforward, numerous network-based methods have been developed to predict novel miRNAs in silico. Homogeneous networks (in which every node is a miRNA) based on the targets shared between miRNAs have been widely used to predict their role in disease phenotypes. Although such homogeneous networks can predict potential disease-associated miRNAs, they do not consider the roles of the target genes of the miRNAs. Here, we introduce a novel method based on a heterogeneous network that not only considers miRNAs but also the corresponding target genes in the network model.

Results: Instead of constructing homogeneous miRNA networks, we built heterogeneous miRNA networks consisting of both miRNAs and their target genes, using databases of known miRNA-target gene interactions. In addition, as recent studies demonstrated reciprocal regulatory relations between miRNAs and their target genes, we considered these heterogeneous miRNA networks to be undirected, assuming mutual miRNA-target interactions. Next, we introduced a novel method (RWRMTN) operating on these mutual heterogeneous miRNA networks to rank candidate disease-related miRNAs using a random walk with restart (RWR) based algorithm. Using both known disease-associated miRNAs and their target genes as seed nodes, the method can identify additional miRNAs involved in the disease phenotype. Experiments indicated that RWRMTN outperformed two existing state-of-the-art methods: RWRMDA, a network-based method that also uses a RWR on homogeneous (rather than heterogeneous) miRNA networks, and RLSMDA, a machine learning-based method. Interestingly, we could relate this performance gain to the emergence of "disease modules" in the heterogeneous miRNA networks used as input for the algorithm. Moreover, we could demonstrate that RWRMTN is stable, performing well when using both experimentally validated and predicted miRNA-target gene interaction data for network construction. Finally, using RWRMTN, we identified 76 novel miRNAs associated with 23 disease phenotypes which were present in a recent database of known disease-miRNA associations.
\end{abstract}

Conclusions: Summarizing, using random walks on mutual miRNA-target networks improves the prediction of novel disease-associated miRNAs because of the existence of "disease modules" in these networks.

Keywords: Disease-associated microRNAs, Network analysis, microRNA targets, Random walk with restart

\footnotetext{
*Correspondence: phamvanhuy@tdt.edu.vn

${ }^{6}$ Faculty of Information Technology, Ton Duc Thang University, Ho Chi Minh

City, Vietnam

Full list of author information is available at the end of the article
} 


\section{Background}

MiRNAs are a class of small non-coding regulatory RNAs that play an important role in the regulation of gene expression $[1,2]$. Misregulation of miRNAs has been shown to contribute to both common [3-7] and rare diseases [8]. Because the identification in the laboratory of miRNAs related to a particular disease is non-trivial, computational methods for the in silico identification of potential disease-miRNAs associations have great potential for speeding up this process.

A number of computational methods, mostly networkbased or machine learning approaches, have been proposed for the prediction of disease-associated miRNAs [9]. The network-based methods mainly rely on the construction of similarity networks expressing functional similarities between miRNAs, after which specific algorithms are used to detect novel disease-miRNA associations [10-20]. Recently, disease similarity matrices have been additionally integrated with the miRNA functional similarity network to construct heterogeneous networks of diseases and miRNAs, using known disease-miRNA associations [21-25].

Most often, the similarity networks used are functional miRNA similarity networks, containing only miRNAs as nodes (hereafter referred to as homogeneous miRNA networks). In these networks, nodes represent miRNAs and edges represent the degree of functional relatedness between the miRNAs. This functional relatedness can be derived from miRNA-target gene interactions in different ways. For example, miRNA functional similarity interactions were constructed based on the degree to which miRNAs share the same targets [10] or by calculating the similarity of target gene regulation patterns for each pair of miRNAs [11]. Additionally, Wang et al. [12] assessed the functional similarity between two miRNAs by comparing the gene functions (using gene ontologies) of their respective sets of target genes. Similarly, $\mathrm{Xu}$ et al. [13] constructed functional synergistic regulatory interactions between miRNAs by considering common target genes in the context of gene ontology and proximity in a protein interaction network. All these methods capture a different aspect of functional similarity, and we demonstrated previously that there can be added value in constructing a functional similarity network by integrating functional similarity interactions obtained using several of the aforementioned methods [14].

Once a homogeneous miRNA networks is available, associations between miRNAs and diseases are subsequently predicted by assuming that functionally related miRNAs associate with phenotypically similar diseases, which is referred to as the "disease module" principle [26, 27]. Specific methods that exploit this principle have been proposed. Local similarity measures only assess direct neighbours of known disease-associated miRNAs [10, 11] or neighbours of candidate miRNAs (as used e.g. by HDMP
[17]) in homogeneous miRNA networks. Another state-ofthe-art method for disease miRNA prediction, RWRMDA $[14,15]$, obtains a global network similarity metric by running a random walk with restart (RWR) algorithm (a network propagation technique) on homogeneous miRNA networks. RWR-based techniques were also applied on different network types where either a phenotype similarity network [20] or a protein interaction network [28] was used as input for the analysis. In addition, we recently demonstrated that network-based ranking algorithms, which were successfully applied for either disease gene prediction or for studying social networks and networks of interlinking web pages, could also be used effectively for disease microRNA prediction on homogeneous miRNA networks, achieving comparable performance with the RWR-based method [16]. For heterogeneous networks of diseases and miRNAs, pathfinding-based methods were used [21, 22] that rely on the assumption that the more paths exist between a miRNA and a disease, the more likely it is that there exists an association between them. In addition, based on the assumption that functionally similar miRNAs tend to be associated with similar diseases, other methods were proposed relying on the identification of clusters of similar diseases and similar miRNAs [23-25].

Next to network-based methods, machine learningbased methods that do not use miRNA-target interactions have also been proposed. For example, a Naïve Bayes model was used to integrate genomic data for prioritizing disease-related miRNAs [29]. Qinghua et al. [30] applied support vector machines for identifying disease-associated miRNAs. In addition, Qabaja et al. [31] used a Lasso regression model to infer disease-miRNA associations. The common limitation of these machine learning methods is the necessity to compile a set of negative training samples consisting of non-disease-related miRNAs. As the absence of an observed association does not imply the non-existence of an association (there are no proven negatives), obtaining such a negative training set is not straightforward [32]. More recently, RLSMDA [33], a semi-supervised classifier-based method, was proposed to overcome this limitation, prioritizing candidate miRNAs for all considered diseases without the need for negative samples. Importantly, RLSMDA was reported to outperform the aforementioned state-of-the-art methods RWRMDA [15] and HDMP [17].

A common limitation of the homogeneous miRNA network-based methods is that the knowledge of biological relationship between miRNAs and their target genes might be used ineffectively because this relationship is only partially integrated in the metric used to capture degree of similarity between two miRNAs. Also, the application of the RWR algorithm, underpinning several state-of-the-art network-based algorithms, is not limited to homogeneous networks containing only miRNA nodes. It 
can be applied to heterogeneous networks where both miRNAs and their gene targets are present in the network as nodes, and edges represent miRNA-target interactions. With the human genome containing thousands of miRNAs $[34,35]$, regulating the expression of thousands of genes [36, 37] and with these miRNA-target interactions (predicted or experimentally validated) now being largely available in a number of miRNA-target databases (as comprehensively reviewed in [38]), here we propose to use heterogeneous networks as input for the identification of disease-related miRNAs, in order to make optimal use of this increased level of detail.

MiRNAs have emerged as key regulators of gene expression in diverse biological pathways; the relationship of a miRNA and its target genes are usually considered as direct interactions between the miRNA and the target genes (i.e., a miRNA regulates target genes by binding to target sequences in mRNAs). Consequently, miRNA-target gene regulatory interactions were used as directed interactions in a number of studies $[32,39,40]$. However, recent developments introduced a new twist to this: targets can reciprocally control the level and function of miRNAs [41]. This mutual regulation of miRNAs and target genes in combination with the large coverage of miRNA-target interactions available in publicly available miRNA-target databases [38] has inspired us to propose a novel network-based method for disease miRNA prediction. In this study, instead of constructing homogeneous miRNA networks from target genes or using directed miRNAtarget gene interactions, we exploit the mutual regulatory relations between miRNAs and their target genes to construct mutual heterogeneous miRNA-target gene networks (hereafter, referred to as mutual heterogeneous miRNA networks). Next, we propose a novel framework, RWRMTN, in which we apply the RWR algorithm on these heterogeneous miRNA networks to prioritize candidate disease miRNAs. In particular, based on a previous study indicating that miRNAs regulate diseases through their target genes [28], we hypothesize that the mutual regulation between a miRNA and their targets leads to a transfer of disease information between them. Therefore, in the proposed method, we force the RWR algorithm to start from a set of seed nodes, consisting not only of known disease miRNAs but also of their target genes. To assess and evaluate the predictive performance of RWRMTN, we use a leave-one-out cross-validation scheme on a set of experimentally verified disease phenotype-miRNA associations. Experimental results indicate that RWRMTN outperforms RWRMDA [15], a state-of-the-art network-based method using RWR operating on homogeneous miRNA networks. Additionally, we demonstrate that this superior performance of our proposed method is because of the existence of "disease modules" in the heterogeneous miRNA networks used as input for our algorithm. Indeed, we observe that (1) a large amount of known disease genes are present in the heterogeneous miRNA networks and (2) most known disease miRNAs in the network regulate at least one known disease gene. Moreover, we showed that our method also outperformed RLSMDA [33], a state-of-the-art machine learning-based method that uses a semi-supervised learning method. Furthermore, we demonstrated that our method is stable and can achieve relative high performance for both experimentally validated and predicted miRNA-target gene interaction data. Finally, using RWRMTN, we identified 76 novel miRNAs associated with 23 disease phenotypes which were present in an recent database of known disease-miRNA associations HMDD [42].

\section{Methods}

\section{Construction of heterogeneous miRNA networks}

To construct heterogeneous miRNA networks, we selected miRWalk [43], a database of experimentally validated miRNA-target interactions and TargetScan [44], a database containing predicted interactions. More specifically, we downloaded experimentally validated human miRNAstarget interactions from the miRWalk database and constructed a heterogeneous miRNA network consisting of 12,721 nodes (745 miRNAs and 11,976 genes) and 38,571 interactions (from now on referred to as HetermiRWal$k N e t$ ) (See in Additional file 1: Table S1). This network can be considered as either a mutual heterogeneous miRNA network (HetermiRWalkNet-mutual) if the interactions between miRNAs and target genes are considered to be reciprocal, or alternatively as a directed heterogeneous miRNA network (HetermiRWalkNet-directed) if miRNAs are assumed to regulate target genes but not vice versa. In addition, we downloaded predicted human miRNA-target gene associations from TargetScan with non-conserved site context++ scores, and constructed a second heterogeneous miRNA network consisting of 16,568 nodes (1547 miRNAs and 15,021 genes) and 520,526 interactions (HeterTargetScanNet) (See in Additional file 1: Table S2). Again, this network can be considered as either a mutual heterogeneous miRNA network (HeterTargetScanNet-mutual) or a directed heterogeneous miRNA network (HeterTargetScanNet-directed). Figure 1a gives an overview of the different types of miRNA networks used in this study.

\section{Construction of homogeneous miRNA networks}

To compare the prediction performance of RWRMTN with that of RWRMDA [15] on homogeneous miRNA networks, we constructed two homogeneous miRNA networks based on miRNA-target gene interactions (Fig. 1b). More specifically, based on an identical procedure of construction of homogeneous miRNA network as in our previous study [16], we defined a functional relation between two miRNAs as follows: two miRNAs are considered to be functionally interacting if they share at least one target 


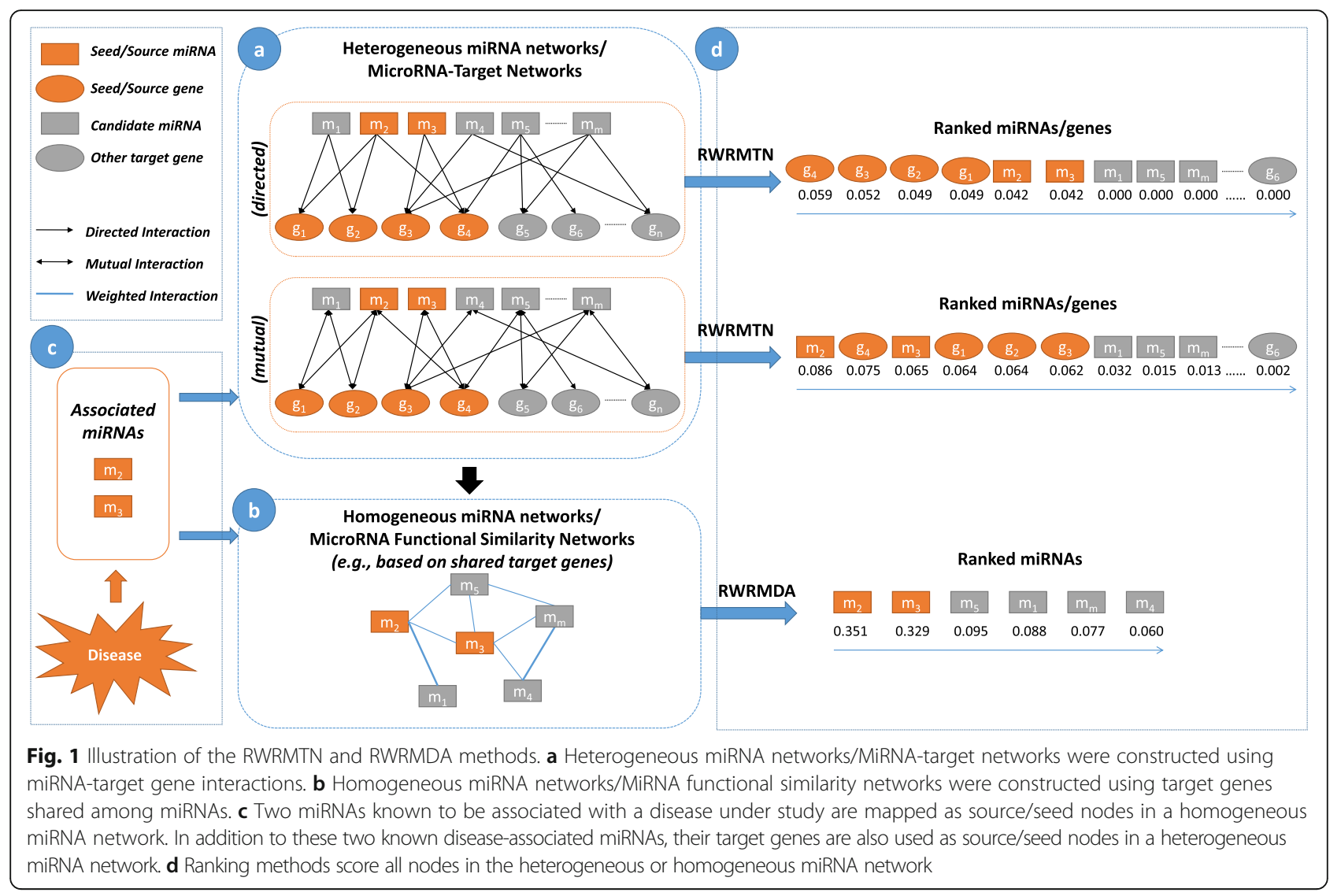

gene, with the degree of similarity defined as the number of shared target genes normalized by the minimum number of target genes of the two miRNAs under consideration. As a result, two networks respectively containing 730 miRNAs with 29,089 interactions (HomomiRWalkNet) and 1428 miRNAs with 46,118 interactions (HomoTargetScanNet) are constructed from the miRNA-target gene interactions in HetermiRWalkNet and HeterTargetScanNet.

\section{Database of known disease phenotype-miRNA associations}

In order to be able to evaluate the performance of the propose method, and to put the new method in perspective, a database of known disease-miRNA associations is required. Here we will use miR2Disease [45], a comprehensive resource of miRNA - human disease associations that is manually curated and maintained. We used 270 manually curated disease phenotype-miRNAs associations between 53 disease phenotypes and 118 miRNAs from that database (See in Additional file 1: Table S3).

\section{Construction of a disease phenotype similarity matrix}

To compare the performance of RWRMTN and RLSMDA, we additionally collected a disease phenotype similarity matrix of 5080 phenotypes from [46], where an element of the matrix represents degree of similarity between two disease phenotypes. The similarities in this matrix were obtained by applying various text mining algorithms to OMIM records [47].

\section{RWRMTN: A random walk with restart algorithm applied to heterogeneous miRNA networks}

RWR is a variant of the random walk algorithm, simulating a walker that either moves from a current node in a network to a randomly selected adjacent node or alternatively returns to the source node (also called the seed node) where the random walk was started, with a fixed probability of returning (restart probability) $\gamma$. This algorithm has been used successfully in a number of related studies such as prediction of disease-associated lncRNA [48], diseaseassociated gene [49], drug target [50] and disease-related microRNA-environmental factor interactions [51].

Given a connected weighted graph $\mathrm{G}(V, E)$ with a set of nodes $V=\left\{v_{1}, v_{2}, \ldots, v_{N}\right\}$ and a set of links $E=\left\{\left(v_{i}, v_{j}\right) \mid\right.$ $\left.v_{\dot{v}} v_{j} \in V\right\}$, a set of seed nodes $S \quad V$, and a $N \times N$ adjacency matrix $W$, the random walk with restart (RWR) can be formally described as follows:

$$
p_{t+1}=(1-\gamma) W^{\prime} p_{t}+\gamma p_{0}
$$

Where $W^{\prime}$ represents a transition probability matrix and $W_{i j}^{\prime}$, the element in $W^{\prime}$ on row $i$ and column $j$, denotes the 
probability that a random walker at node $v_{i}$ moves to neighboring node $v_{j}$ :

$$
W_{i j}^{\prime}=\frac{W_{i j}}{\sum_{k \in\left(V_{\text {out }}\right)_{i}} W_{i k}}
$$

Here

- $\left(V_{\text {out }}\right)_{i}$ is a set of outgoing nodes of $v_{i}$. If an unweighted graph (e.g., a heterogeneous miRNA network) is used, all interactions are assigned a unity weight.

- $p_{\mathrm{t}}$ is a $N \times 1$ probability vector of $|V|$ nodes at a time step $t$ of which the $i^{\text {th }}$ element represents the probability of the walker being at node $v_{i} \in V$.

- $p_{0}$ is the $N \times 1$ initial probability vector.

In the RWRMDA method, the RWR technique is used to rank miRNAs in homogeneous miRNA networks. Therefore, the set of seed nodes $S$ only contains known disease miRNAs (i.e., $S=S_{m}$ ) and $p_{0}$ is defined as follows:

$$
\left(p_{0}\right)_{i}= \begin{cases}\frac{1}{\left|S_{m}\right|} & i f v_{i} \in S_{m} \\ 0 & \text { otherwise }\end{cases}
$$

Alternatively, for RWRMTN we assume that the mutual regulation between a miRNA and their targets leads to an exchange of disease information between the two entities participating in the interaction. Therefore, we enlarge the set of seed node $S$ by adding target genes $S_{g}$ of the known disease miRNAs (i.e., $S=S_{m} \cup S_{g}$ ). The initial probability vector $p_{O}$ is defined as follows:

$$
\left(p_{0}\right)_{i}=\left\{\begin{array}{cc}
\alpha \frac{1}{\left|S_{m}\right|} & i f v_{i} \in S_{m} \\
(1-\alpha) \frac{1}{\left|S_{g}\right|} & \text { ifviv } v_{g} \\
0 & \text { otherwise }
\end{array}\right.
$$

where $\alpha \in[0,1]$ is a weight parameter, controlling the amount of disease information transferred between miRNAs and their target genes.

For both methods, all miRNAs/genes in the network are eventually ranked according to the steady-state probability vector $p_{\infty}$, which is obtained by repeating the iterations until convergence is reached (in this study, ||$\left.p_{\mathrm{t}+1}-p_{\mathrm{t}} \|<10^{-6}\right)$.

Note that, for directed heterogeneous miRNA networks such as HetermiRWalkNet-directed and HeterTargetScanNet-directed, the random walker is trapped at seed target genes because there is no outgoing link at these nodes. Therefore, non-seed nodes (including previously unidentified disease miRNAs and other target genes) cannot be ranked as they are all assigned a zero probability (Fig. 1d). Therefore, RWRMTN can only be applied to mutual heterogeneous miRNA networks such as HetermiRWalkNet-mutual and HeterTargetScanNetmutual. Figure 1 illustrates these two methods.

\section{RLSMDA: Regularized least squares for MiRNA-disease association}

RLSMDA is a semi-supervised and global method since it can rank disease-miRNA associations for all diseases under consideration simultaneously, without the need for a negative training set. RLSMDA constructs a continuous function that can determine the association probability between each miRNA and a given disease. The higher this probability is, the more a miRNA is related to a given disease. To this end, RLSMDA relies on the minimization of two cost functions, defined in respectively the miRNA space and in the disease space, whose solutions are subsequently combined in a single continuous classification function [33]. The optimal classifier in these two spaces was defined as follows:

$$
F^{*}=w F_{M}^{* T}+(1-w) F_{D}^{*}
$$

where $F_{M}^{*}$ and $F_{D}^{*}$ are optimal classification functions in the miRNA and disease phenotype spaces, respectively defined as:

$$
\begin{aligned}
& F_{M}^{*}=S_{M}\left(S_{M}+\eta_{M} I_{M}\right) A^{T} \\
& F_{D}^{*}=S_{D}\left(S_{D}+\eta_{D} I_{D}\right) A
\end{aligned}
$$

with

- $w$ is the weight between these two spaces. $\eta_{M}$ and $\eta_{D}$ are trade-off parameters in the miRNA and disease phenotype spaces, respectively.

- $S_{D}(m \times m)$ is the disease phenotype similarity matrix containing $m$ diseases. $S_{M}(n \times n)$ is the corresponding similarity matrix of the homogeneous miRNA network containing $n$ miRNAs, where $S_{M}(i$, $j$ ) is the degree of similarity between two miRNAs.

- $I_{M}$ and $I_{D}$ are identity matrices with the same size as matrices $S_{M}$ and $S_{D}$, respectively.

- $A(m \times n)$ is an association matrix, where $A(i, j)=1$ if disease phenotype $i$ is known to be associated with miRNA $j$, otherwise $A(i, j)=0$.

\section{Performance evaluation}

To compare the potential of RWRMTN for associating novel miRNAs with disease phenotypes with that of RWRMDA and RLSMDA, we applied a leave-one-out cross-validation (LOOCV) scheme on the set of disease phenotypes with known miRNA associations in miR2Disease [45]. For each disease phenotype $d$, in each round of LOOCV, we held out one known miRNA associated 
with $d$. The rest of the known miRNAs associated with disease $d$ are used as seed nodes $\left(S_{m}\right)$ in the RWRMDA method. For the RWRMTN method, this set was enlarged by adding the target genes $S_{g}$ of the miRNAs in $S_{m}$. The held-out miRNA and the remaining miRNAs in the miRNA networks which were not known to be associated with $d$, were ranked by both RWRMTN and RWRMDA. For RLSMDA, $A(i, j)$ is set to 0 corresponding to $d$ and the held-out miRNA. Then, receiver operating characteristic (ROC) curves are constructed and the area under the curve (AUC) is used to compare the performance of both methods. The ROC curve represents the relationship between sensitivity and (1-specificity), where sensitivity refers to the percentage of miRNAs known to be associated with $d$ that were ranked above a particular threshold and specificity refers to the percentage of miRNAs that were not known to be associated with $d$ and ranked below this threshold. Finally, the performance of each method was summarized as the average of AUC values over the entire set of disease phenotypes in the validation set.

\section{Results and discussion}

\section{Parameter settings}

To determine the best setting for RWRMTN, we varied the weight parameter $(\alpha)$ in the range $\{0.1,0.3,0.5,0.7$, $0.9\}$ and the restart probability $\gamma$ in the range $[0.1,0.9]$ in steps of 0.1 . For each combination of parameter values, we only assessed the performance of RWRMTN on mutual heterogeneous miRNA networks as the method cannot be applied to directed heterogeneous miRNA networks (See Materials and Methods). Performance was assessed as the average AUC over the set of disease phenotypes in the disease phenotype set (See Materials and Methods). Fig. 2a and b shows that the performance of RWRMTN slightly increased according to the change of the weight parameter on mutual heterogeneous miRNA networks constructed from miRWalk (HetermiRWalkNet- mutual) and from TargetScan (HeterTargetScanNet-mutual). This indicates that disease information contained in known disease miRNAs is still more important than that in their target genes when prioritizing candidate diseaseassociated miRNAs. In addition, optimal performance was achieved for both networks with $\alpha=0.9$ and $\gamma=0.7$. For the RLSMDA method, we used the parameter settings $\left(\eta_{M}=\eta_{D}=1, w=0.9\right)$ reported in the corresponding study [33].

\section{Performance comparison}

In this section, we compare the performance of RWRMTN with two state-of-the-art methods. We selected RWRMDA [15] as a representative network-based method, as we intended to demonstrate the added value of using heterogeneous miRNA networks over using homogeneous miRNA networks. Additionally we compared with RLSMDA [33], a state-of-the-art machine learning-based method, that does not use a network as a basis for its analysis.

\section{Comparison between RWRMTN and RWRMDA}

In a previous study [16], we demonstrated that other homogeneous miRNA network-based methods achieve performance similar to RWRMDA [15], a RWR-based method. Therefore, in this study, we only compare the prediction performance of RWRMTN on the heterogeneous miRNA networks with that of RWRMDA on the homogeneous miRNA networks. More specifically, we tested the performance of RWRMTN on the two mutual heterogeneous miRNA networks, HetermiRWalkNet-mutual and HeterTargetScanNet-mutual, and the performance of RWRMDA on the two homogeneous miRNA networks, HomomiRWalkNet and HomoTargetScanNet. In all experiments, we varied the random walker's restart probability $\gamma$ in a range of $[0.1,0.9]$ for both methods, and set the weight parameter $\alpha$ of RWRMTN to 0.9. The performance of both methods on each heterogeneous/homogeneous miRNA network is expressed as the average AUC values over the a

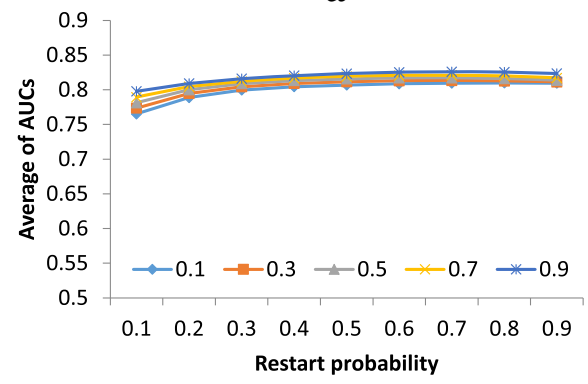

b

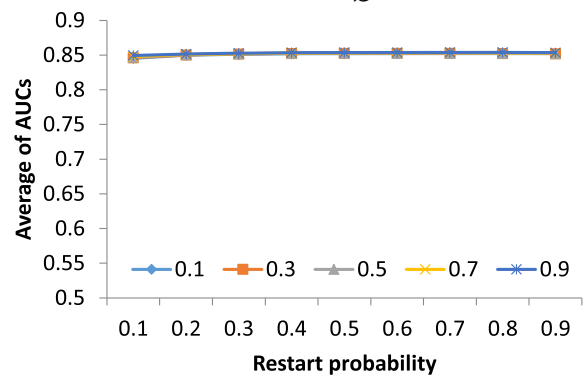

Fig. 2 Performance of RWRMTN as a function of the algorithm parameters, using mutual heterogeneous miRNA networks. Performance is an average of AUC values over a set of disease phenotypes collected from the miR2Disease database [45]. The restart probability $\gamma$ was varied in the range $[0.1,0.9]$. The weight parameter a) was set to values in $\{0.1,0.3,0.5,0.7,0.9\}$. Results are reported for (a) HetermiRWalkNet-mutual and (b) HeterTargetScanNet-mutual 
set of available disease phenotypes. Figure 3 shows the prediction performance of the two methods on heterogeneous/homogeneous miRNA networks constructed from miRWalk and TargetScan databases respectively. Analyzing the performance of the two methods on different heterogeneous/homogeneous miRNAs networks, we observed that the performance of RWRMDA on HomomiRWalkNet and HomoTargetScanNet was respectively slightly better and stable when the restart probability $\gamma$ increased (the slopes of regression line are respectively 0.045 and -0.006 with $p$ $=0.001$ and $p=0.239$, Fig. 3). This difference in performance response to the restart probability (increase vs. stable) when using different networks as input can be explained by the fact that when the restart probability is small, the random walker is able to travel relatively far from the seed nodes. This in turn allows for an improved exploitation of the "disease module" principle since it tends to assign higher scores to nodes close to the seed nodes. Therefore, the stable performance of RWRMDA as a function of the restart probability on homogeneous miRNA networks suggests that disease miRNAs are relatively close or directly connected to each other in the individual homogeneous miRNA networks. The increase in performance (when varying $\gamma$ ) observed when using HomomiRWalkNet suggests that disease miRNAs in this network are less modularized than those in HomoTargetScanNet.

In contrast to the homogeneous miRNA networks, miRNAs connect to each other via target genes in the heterogeneous miRNA networks. In other words, disease miRNAs are less modularized in these networks. Indeed, Fig. 3 show that the performance of RWRMTN slightly increased when the restart probability increased in both networks (the slopes of regression lines are 0.029 and 0.004 with $p=0.004$ and $p=0.011$, respectively for HetermiRWalkNet-mutual and HeterTargetScanNet-mutual). It is also slightly more positive on HetermiRWalkNet-mutual indicating that disease miRNAs/genes in that network is less modularized than those in HeterTargetScanNet-mutual.
Interestingly, the performance of RWRMTN on HetermiRWalkNet-mutual and HeterTargetScanNet-mutual is consistently higher than that of RWRMDA on HomomiRWalkNet and HomoTargetScanNet (two sample t-Test, $p$ $=1.24 \times 10^{-6}$ and $7.59 \times 10^{-9}$, respectively). Average AUC values of RWRMTN on HetermiRWalkNet-mutual and HeterTargetScanNet-mutual are 0.819 and 0.853 . Average AUC values of RWRMDA on HomomiRWalkNet and HomoTargetScanNet are 0.776 and 0.830 . These results suggest that using mutual biological relations between miRNAs and their target genes helps improving the disease miRNA prediction. In other words, information contained in these biological relations is used less effectively when it is integrated as the degree of similarity between miRNAs in the homogeneous miRNA networks. In addition, the "disease module" idea can be expected to be more explicitly present in the heterogeneous miRNA networks. This principle is generally accepted for both miRNAs (functionally related miRNAs associate with phenotypically similar diseases [26, 27]) and genes (functionally related genes associate with phenotypically similar diseases [52-54]). Two miRNAs in a heterogeneous miRNA network are functionally related if they regulate the same target genes, but conversely, we can assume that two genes regulated by the same miRNAs can be functionally related too. To illustrate this, we investigated how many known disease genes are present as targets of miRNAs in our heterogeneous miRNA networks. We downloaded disease-gene associations from OMIM at the NCBI website [55] and retrieved 4388 associations between 3.284 disease phenotypes and 2,761 disease genes. Figure $4 \mathrm{a}$ and $\mathrm{b}$ shows that from these disease genes, $1,855 \quad(\sim 67.19 \%)$ and $2,262 \quad(\sim 81.93 \%)$ known disease genes are found as target genes in the heterogeneous miRNA networks respectively built from miRWalk and TargetScan. This implies that a large amount of disease genes are regulated by miRNAs. In addition, we investigated how many known disease miRNAs regulate
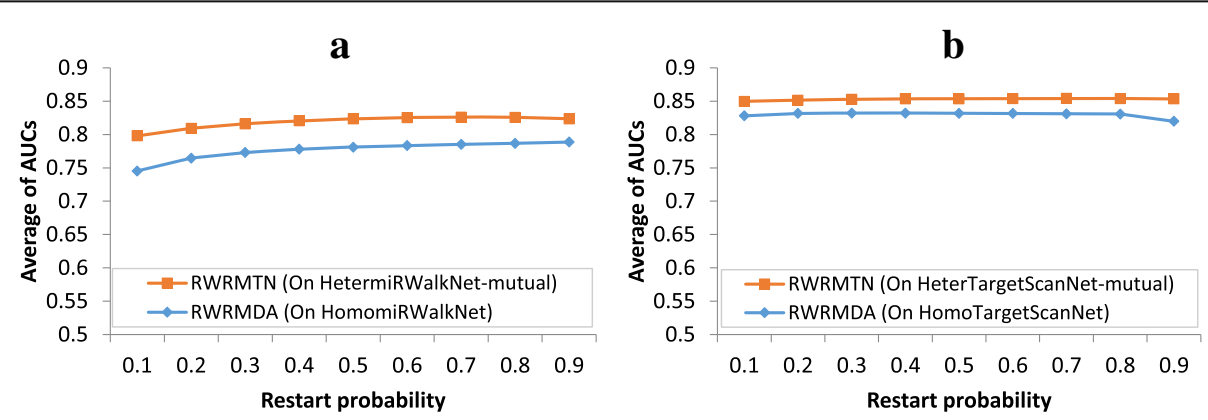

Fig. 3 Performance comparison between RWRMTN and RWRMDA. The performance of each method on each heterogeneous/homogeneous miRNA network is calculated as the average AUC values over a set of disease phenotypes collected from the miR2Disease database [45]. The restart probability was varied from 0.1 to 0.9. The weight parameter was set to 0.1. a Comparison between RWRMTN (using HetermiRWalkNet-mutual) and RWRMDA (using HomomiRWalkNet). b Comparison between RWRMTN (using HeterTargetScanNet-mutual) and RWRMDA (using HomoTargetScanNet) 

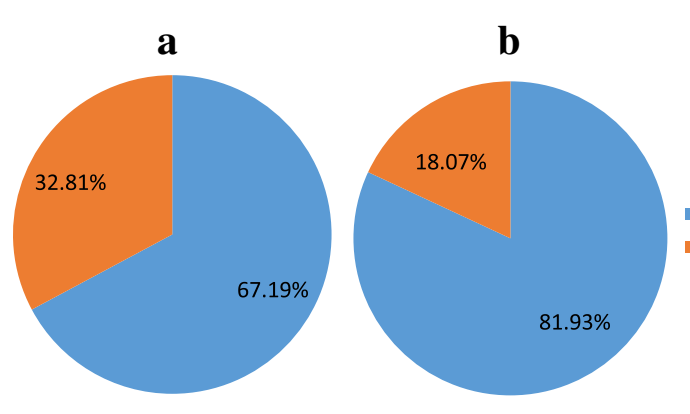

- Known disease genes present in the network

Known disease genes absent in the network
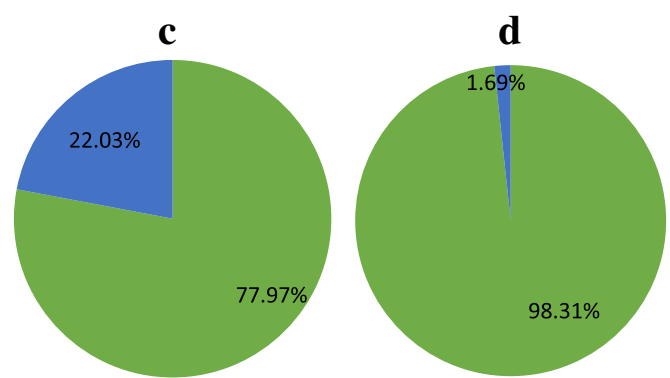

Known disease miRNAs that regulate at least one known disease gene

- Known disease miRNAs that don't regulate any known disease gene

Fig. 4 Heterogeneous miRNA networks contain known disease genes and known disease miRNAs, regulating known disease genes. a Percent of known disease genes in HetermiRWalkNet-mutual. b Percent of known disease genes in HeterTargetScanNet-mutual. c Percent of known disease miRNAs regulating disease genes in HetermiRWalkNet-mutual. $\mathbf{d}$ Percent of known disease miRNAs regulating disease genes in HeterTargetScanNet-mutual. Known disease genes and known disease miRNAs were collected from the OMIM [47] and miR2Disease [45] databases, respectively

known disease genes in the heterogeneous miRNA networks. Figure $4 \mathrm{c}$ and d shows that $92(\sim 77.97 \%)$ and 116 ( 98.31\%) out of 118 known disease miRNAs (see Materials and Methods) regulate at least one known disease gene in the heterogeneous miRNA networks constructed from the miRWalk and TargetScan databases. This indicates that a large amount of disease miRNAs regulate disease genes. The smaller fraction of known disease miRNAs found in HetermiRWalkNet-mutual compared to that in HeterTargetScanNet-mutual also indicates that disease miRNAs/genes in the former is less modularized compared to those in the later. Taken together, these results imply that disease-associated miRNAs and genes are located closely to each other in the heterogeneous networks. Therefore, considering them together by using heterogeneous miRNA networks when predicting novel disease-associated miRNAs can be advantageous.

\section{Comparison between RWRMTN and RLSMDA}

In addition to comparing with a representative networkbased method, we also compared our method with RLSMDA [33], a state-of-the-art machine learning-based technique. To this end, we used the optimal set of parameters ( $\alpha=0.9$ and $\gamma=0.7)$ for RWRMTN as obtained in the previous experiment. For RLSMDA, we used the parameter settings $\left(\eta_{M}=\eta_{D}=1\right.$ and $\left.w=0.9\right)$ reported in the corresponding study [33]. Again, we used the ROC and AUC to compare these two methods on different databases of miRNA-target interactions. Figure 5 illustrates that RWRMTN (average AUCs are 0.826 and 0.854 in
HetermiRWalkNet and HeterTargetScanNet respectively) outperforms RLSMDA (average AUCs are 0.757 and 0.795 in HomomiRWalkNet and HomoTargetScanNet respectively), suggesting that the explicit use of gene-miRNA interactions has an added value when predicting novel disease-related miRNAs. Comparing RWRMDA with RLSMDA, we used the best settings for RWRMDA and found the average AUCs of RWRMDA to be $0.789(\gamma=$ $0.9)$ and $0.832(\gamma=0.3)$ in HomomiRWalkNet and HomoTargetScanNet respectively. This indicates that using functional miRNA interactions in RWRMDA results in inferior predictions compared to using miRNA-gene interactions in RWRMTN, but these predictions still outperform RLSMDA where no explicit network information is used.

\section{Comparison between RWRMTN and RWRMDA, RLSMDA using 10-fold cross-validation}

In previous section, we compare the performance of RWRMTN with that of RWRMDA and RLSMDA using leave-one-out cross validation (LOOCV). Considering that LOOCV is equivalent to $\mathrm{n}$-fold cross validation (where $\mathrm{n}$ is number of known miRNAs of a given disease), this evaluation method is flexible and can be used to assess the prediction performance for any disease, even for those with only two known associated miRNAs. To show the robustness and stability of our method, we further test it with 10 -fold cross validation on the TargetScan database. Due to this re-sampling method, only diseases known to be associated with at least 10 miRNAs can be taken into account. Using this criterion, 

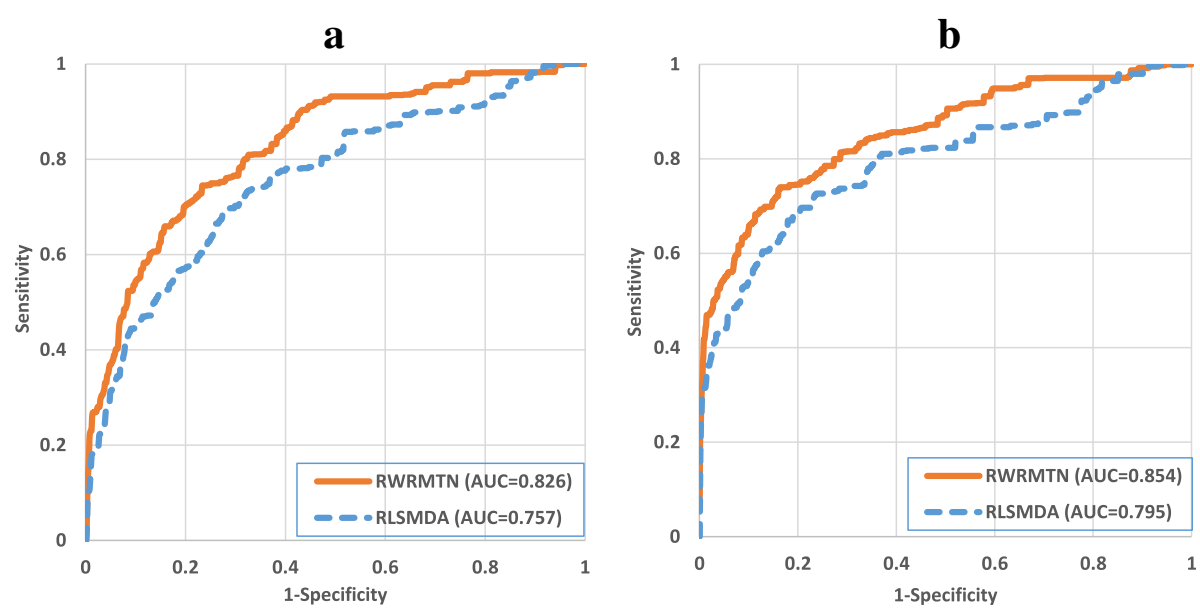

Fig. 5 Comparison between RWRMTN and RLSMDA. The set of disease phenotypes and their associated miRNAs were collected from the miR2Disease database [45]. a MiRNA networks were constructed using the miRWalk database. b MiRNA networks were constructed using TargetScan database. Weight parameter $a$ and restart probability $\gamma$ were set to the optimal settings ( $a=0.9$ and $\gamma=0.7)$ for RWRMTN. For RLSMDA, we used the parameter settings $\left(\eta_{M}=\eta_{D}=1\right.$ and $\left.w=0.9\right)$ reported in the study [33]

only eight diseases in miR2Disease [45] were found to be eligible for validation. Additional file 2: Figure S1 shows the performance of the three methods using their respective optimal parameter settings (i.e., $\alpha=0.9$ and $\gamma$ $=0.7$ for RWRMTN, $\gamma=0.7$ for RWRMDA, and $\eta_{M}=\eta_{D}$ $=1, w=0.9$ for RLSMDA). It is obvious that RWRMTN $(\mathrm{AUC}=0.840)$ outperforms both RWRMDA (AUC = 0.792) and RLSMDA (AUC $=0.753$ ). We additionally used a larger disease-miRNA association database HMDD (version 2.0 [42]), containing 57 diseases eligible for performance assessment using 10 -fold cross validation. Additional file 2: Figure S2 indicates that, again with optimal parameter settings for each method, the performance of RWRMTN (AUC = 0.896) is better than that observed for both RWRMDA (AUC $=0.875)$ and RLSMDA (AUC $=0.749$ ).

\section{Identification of novel disease-associated miRNAs}

To illustrate the power of RWRMTN to identify novel disease-associated miRNAs, we next tried to predict newly reported disease miRNAs in the experimentally verified disease-miRNA association HMDD database (version 2.0 [42]). As input for this analysis, we used known disease miRNAs as reported in the miR2Disease database [45]. First, we selected 23 disease phenotypes that were available in both databases. Then, for each disease phenotype, we used known associated miRNAs (as reported in the miR2Disease database) and their target genes as seed nodes in the HeterTargetScanNet-mutual network. We used the optimal parameter settings identified in the previous experiments $(\alpha=0.9$ and $\gamma=0.7)$ and ran our method to rank all remaining miRNAs in the network. After ranking, we selected the 100 top-ranked candidate miRNAs for each disease phenotype and checked whether they were reported in HMDD. Table 1 shows the results of this analysis. In total, 76 distinct novel disease miRNAs were predicted for the 23 disease phenotypes. We further tested per disease whether the selected 100 miRNAs were significant enriched for miRNAs reported in HMDD using a hypergeometric test [56]. For 18 out of the 23 disease phenotypes, we found the enrichment of the 100 predicted miRNAs for miRNAs reported in HMDD to be statistically significant $(\mathrm{p} \leq 0.05)$. The remaining highly ranked miRNAs, for which no evidence about the association with the considered disease phenotypes yet exists, are candidates for further exploration in future studies (See in Additional file 1: Table S4). For several diseases, no significant enrichment of disease related miRNAs could be found. As Table 1 illustrates, this is due to the very small number of miRNAs that were associated with these diseases in the HMDD database. However, our topranked predictions contained miRNAs that have previously been associated with a disease, even though these associations were not present in HMDD. For example, hsa-miR-137 regulates the expression of the HTT gene, whose mutation leads to Huntington's disease [57]. hsa-miR-15a and hsa-miR-27a are involved in human adipocyte differentiation and obesity [58]. Nicholas et al. [59] found that exposure to maternal obesity resulted in increased hepatic hsa-miR-29b. While investigating kidney tissue, which is known to be invoked in the etiology of essential hypertension, hsa-miR-181a and hsa-let-7c were found to be differentially expressed between kidneys of 15 untreated hypertensive and 7 normotensive white male subjects [60]. hsa-miR-181b and hsa-miR-181d were found to be differentially expressed between invasive and noninvasive non-functional pituitary adenoma [61]. Finally, 
Table 1 MiRNAs present in the top-100 ranked candidate miRNAs that are known to be associated with diseases, as reported in the HMDD database. P-value is the result of the hypergeometric enrichment test

\begin{tabular}{|c|c|c|c|c|c|}
\hline MIM ID & Disease & Overlap & $\begin{array}{l}\text { Total in } \\
\text { HMDD }\end{array}$ & $p$-value & Known disease miRNAs \\
\hline 150699 & leiomyoma & 2 & 3 & 0.012 & hsa-miR-106b, hsa-miR-93 \\
\hline 109800 & bladder cancer & 6 & 27 & 0.006 & hsa-miR-17, hsa-miR-182, hsa-miR-200b, hsa-miR-200c, hsa-miR-20a, hsa-miR-27a \\
\hline 143100 & huntington disease & 1 & 7 & 0.376 & hsa-miR-200c \\
\hline 601665 & obesity & 2 & 7 & 0.091 & hsa-miR-17, hsa-miR-30e \\
\hline 145500 & hypertension & 3 & 15 & 0.069 & hsa-let-7e, hsa-miR-17, hsa-miR-20a \\
\hline 600634 & pituitary adenoma & 1 & 14 & 0.065 & hsa-miR-107 \\
\hline 133239 & esophageal cancer & 8 & 51 & 0.017 & $\begin{array}{l}\text { hsa-let-7a, hsa-let-7b, hsa-let-7c, hsa-miR-19a, hsa-miR-200c, hsa-miR-203, hsa-miR-29c, } \\
\text { hsa-miR-98 }\end{array}$ \\
\hline 181500 & schizophrenia & 17 & 29 & $2.19 \times 10^{-13}$ & $\begin{array}{l}\text { hsa-miR-106b, hsa-miR-137, hsa-miR-15a, hsa-miR-15b, hsa-miR-17, hsa-miR-181b, hsa-miR-195, } \\
\text { hsa-miR-20b, hsa-miR-26b, hsa-miR-29a, hsa-miR-29b, hsa-miR-29c, hsa-miR-30a, hsa-miR-30b, } \\
\text { hsa-miR-30d, hsa-miR-30e, hsa-miR-9 }\end{array}$ \\
\hline 603956 & cervical cancer & 2 & 3 & 0.023 & hsa-miR-20a, hsa-miR-424 \\
\hline 155601 & melanoma & 34 & 130 & $8.97 \times 10^{-14}$ & $\begin{array}{l}\text { hsa-let-7c, hsa-let-7d, hsa-let-7e, hsa-let-7f, hsa-let-7 g, hsa-let-7i, hsa-miR-106a, hsa-miR-106b, } \\
\text { hsa-miR-137, hsa-miR-15a, hsa-miR-15b, hsa-miR-16, hsa-miR-17, hsa-miR-181a, hsa-miR-182, } \\
\text { hsa-miR-195, hsa-miR-196a, hsa-miR-19a, hsa-miR-19b, hsa-miR-200b, hsa-miR-200c, } \\
\text { hsa-miR-20a, hsa-miR-20b, hsa-miR-218, hsa-miR-23b, hsa-miR-27b, hsa-miR-30a, } \\
\text { hsa-miR-30b, hsa-miR-30d, hsa-miR-30e, hsa-miR-429, hsa-miR-506, hsa-miR-9, hsa-miR-93 }\end{array}$ \\
\hline 151400 & leukemia & 6 & 26 & 0.009 & hsa-miR-17, hsa-miR-181a, hsa-miR-19a, hsa-miR-19b, hsa-miR-20a, hsa-miR-27a \\
\hline 268210 & rhabdomyosarcoma & 2 & 7 & 0.112 & hsa-miR-106a, hsa-miR-29a \\
\hline 104300 & alzheimer disease & 10 & 16 & $9.36 \times 10^{-8}$ & $\begin{array}{l}\text { hsa-miR-106b, hsa-miR-124, hsa-miR-125b, hsa-miR-128, hsa-miR-137, hsa-miR-17, hsa-miR-181c, } \\
\text { hsa-miR-195, hsa-miR-20a, hsa-miR-9 }\end{array}$ \\
\hline 256700 & neuroblastoma & 10 & 29 & $1.23 \times 10^{-5}$ & $\begin{array}{l}\text { hsa-miR-106b, hsa-miR-124, hsa-miR-128, hsa-miR-19a, hsa-miR-19b, hsa-miR-20a, hsa-miR-27b, } \\
\text { hsa-miR-340, hsa-miR-9, hsa-miR-93 }\end{array}$ \\
\hline 113970 & burkitt lymphoma & 5 & 10 & $2.05 \times 10^{-4}$ & hsa-miR-17, hsa-miR-19a, hsa-miR-19b, hsa-miR-20a, hsa-miR-93 \\
\hline 114500 & colorectal cancer & 26 & 120 & $3.04 \times 10^{-8}$ & $\begin{array}{l}\text { hsa-let-7b, hsa-let-7c, hsa-let-7e, hsa-miR-106a, hsa-miR-137, hsa-miR-17, hsa-miR-181a, } \\
\text { hsa-miR-181b, hsa-miR-182, hsa-miR-195, hsa-miR-19a, hsa-miR-19b, hsa-miR-200b, hsa-miR-200c, } \\
\text { hsa-miR-20a, hsa-miR-218, hsa-miR-23a, hsa-miR-26a, hsa-miR-26b, hsa-miR-27b, hsa-miR-29a, } \\
\text { hsa-miR-340, hsa-miR-497, hsa-miR-9, hsa-miR-93, hsa-miR-96 }\end{array}$ \\
\hline 260350 & pancreatic cancer & 23 & 89 & $6.42 \times 10^{-9}$ & $\begin{array}{l}\text { hsa-let-7b, hsa-let-7c, hsa-let-7d, hsa-let-7e, hsa-let-7f, hsa-let-7 g, hsa-let-7i, hsa-miR-106a, } \\
\text { hsa-miR-128, hsa-miR-15a, hsa-miR-15b, hsa-miR-17, hsa-miR-181b, hsa-miR-182, hsa-miR-200a, } \\
\text { hsa-miR-200c, hsa-miR-20a, hsa-miR-23a, hsa-miR-26a, hsa-miR-27a, hsa-miR-30c, hsa-miR-429, } \\
\text { hsa-miR-96 }\end{array}$ \\
\hline 211980 & lung cancer & 26 & 96 & $5.79 \times 10^{-9}$ & $\begin{array}{l}\text { hsa-let-7i, hsa-miR-106a, hsa-miR-181a, hsa-miR-181b, hsa-miR-181c, hsa-miR-182, hsa-miR-19b, } \\
\text { hsa-miR-200b, hsa-miR-200c, hsa-miR-206, hsa-miR-23a, hsa-miR-25, hsa-miR-27b, hsa-miR-301a, } \\
\text { hsa-miR-30a, hsa-miR-30b, hsa-miR-30c, hsa-miR-30d, hsa-miR-30e, hsa-miR-32, hsa-miR-497, } \\
\text { hsa-miR-9, hsa-miR-92a, hsa-miR-93, hsa-miR-96, hsa-miR-98 }\end{array}$ \\
\hline 168600 & parkinson disease & 7 & 24 & $9.43 \times 10^{-4}$ & hsa-miR-19b, hsa-miR-29a, hsa-miR-29b, hsa-miR-29c, hsa-miR-30a, hsa-miR-30b, hsa-miR-30c \\
\hline 114480 & breast cancer & 41 & 170 & $1.96 \times 10^{-13}$ & $\begin{array}{l}\text { hsa-let-7b, hsa-let-7c, hsa-let-7d, hsa-let-7e, hsa-let-7f, hsa-let-7 g, hsa-let-7i, hsa-miR-1, } \\
\text { hsa-miR-106b, hsa-miR-137, hsa-miR-15a, hsa-miR-16, hsa-miR-181a, hsa-miR-181b, hsa-miR-182, } \\
\text { hsa-miR-195, hsa-miR-19a, hsa-miR-19b, hsa-miR-202, hsa-miR-20b, hsa-miR-23a, hsa-miR-23b, } \\
\text { hsa-miR-27b, hsa-miR-29a, hsa-miR-29b, hsa-miR-29c, hsa-miR-302a, hsa-miR-302b, hsa-miR-302c, } \\
\text { hsa-miR-302d, hsa-miR-30a, hsa-miR-30b, hsa-miR-30c, hsa-miR-30d, hsa-miR-340, hsa-miR-497, } \\
\text { hsa-miR-519d, hsa-miR-520b, hsa-miR-9, hsa-miR-93, hsa-miR-96 }\end{array}$ \\
\hline 236000 & lymphoma & 14 & 47 & $5.64 \times 10^{-7}$ & $\begin{array}{l}\text { hsa-miR-124, hsa-miR-133b, hsa-miR-15a, hsa-miR-17, hsa-miR-181a, hsa-miR-19a, hsa-miR-19b, } \\
\text { hsa-miR-200b, hsa-miR-200c, hsa-miR-20a, hsa-miR-20b, hsa-miR-218, hsa-miR-26a, hsa-miR-29c }\end{array}$ \\
\hline 155255 & medulloblastoma & 9 & 57 & 0.013 & $\begin{array}{l}\text { hsa-miR-106a, hsa-miR-17, hsa-miR-181b, hsa-miR-182, hsa-miR-19a, hsa-miR-19b, hsa-miR-20a, } \\
\text { hsa-miR-30a, hsa-miR-96 }\end{array}$ \\
\hline 137215 & gastric cancer & 27 & 123 & $3.56 \times 10^{-9}$ & $\begin{array}{l}\text { hsa-let-7f, hsa-let-7 g, hsa-miR-106a, hsa-miR-107, hsa-miR-124, hsa-miR-130a, hsa-miR-17, } \\
\text { hsa-miR-181a, hsa-miR-181b, hsa-miR-182, hsa-miR-195, hsa-miR-200b, hsa-miR-200c, } \\
\text { hsa-miR-20a, hsa-miR-27a, hsa-miR-27b, hsa-miR-29a, hsa-miR-30b, hsa-miR-30c, hsa-miR-340, } \\
\text { hsa-miR-372, } \\
\text { hsa-miR-373, hsa-miR-429, hsa-miR-497, hsa-miR-503, hsa-miR-519a, hsa-miR-9 }\end{array}$ \\
\hline
\end{tabular}


hsa-let-7c-5p facilitated enterovirus 71 replication through viral subversion of cell signaling in rhabdomyosarcoma cells [62].

\section{Conclusions}

MiRNAs are known to have a strong impact on biological processes and play a pathogenic role in human diseases [63]. Therefore, the identification of novel disease-associated miRNAs is an essential part of biomedical research studying the underlying mechanisms of human diseases. Here we proposed a novel approach using a random walk with restartbased algorithm applied on mutual heterogeneous miRNA networks (RWRMTN), where contrary to previous efforts, miRNA-target gene relations were considered as bidirectional interactions, and the network used as input explicitly incorporates miRNA-target interactions. Experimental results demonstrate that our method achieves higher performance than a state-of-the-art network-based method (RWRMDA) that uses homogeneous miRNA networks, only containing miRNA nodes. We motivated that the superior performance of the proposed method can be partially attributed to the improved exploitation of the "disease module" principle. This concept is explicitly present in the heterogeneous miRNA networks used as input for our analysis, and we showed that a large amount of disease-associated miRNAs and disease related genes mutually interact with each other. Additionally, our method outperformed RLSMDA [33], a stateof-the-art machine learning-based method, confirming the added value of using network information when predicting novel disease related miRNAs. MiRNA-target interaction data predicted by in silico prediction tools typically have a high rate of false positive and false negative results. Therefore, we applied our method to two databases containing respectively predicted and experimentally validated miRNAs-target interactions. We could show that our method can achieve stable and high performance for both experimentally validated and predicted interaction data. Finally, using RWRMTN, we identified 76 miRNAs which were reported to be associated with 23 disease phenotypes in HMDD, an recent disease-miRNA association database.

\section{Additional files}

Additional file 1: Table S1. A heterogeneous miRNA network constructed from the miRWalk database. Table S2. A heterogeneous miRNA network constructed from the TargetScan database. Table S3. List of disease phenotypes and their known associated miRNAs, as collected from the miR2Disease database. Table S4. miRNAs in the sets of 100 top-ranked candidate miRNAs that are not known to be associated with diseases in the HMDD database. (XLSX 7368 kb)

Additional file 2: Figure S1. Performance comparison between RWRMTN, RWRMDA and RLSMDA on miR2Disease database using 10-fold cross validation. Figure S2. Performance comparison between RWRMTN, RWRMDA and RLSMDA on HMDD database using 10-fold cross validation. (DOCX $185 \mathrm{~kb})$
Acknowledgements

Not applicable.

\section{Funding}

This research is funded by Foundation for Science and Technology Development of Ton Duc Thang University (FOSTECT), website: http:// fostect.tdt.edu.vn, under Grant FOSTECT.2016.BR.09.

\section{Availability of data and materials}

Source code and experiment data can be accessed at https:// sites.google.com/site/duchaule2011/bioinformatics-tools/rwrmtn

\section{Authors' contributions}

DHL conceived of the study, DHL wrote the program code. DHL, LV, LHS, DTC and VHP wrote the manuscript. All authors read and approved the final version of the manuscript.

Ethics approval and consent to participate

Not applicable.

Consent for publication

Not applicable.

Competing interests

The authors declare that they have no competing interests.

\section{Publisher's Note}

Springer Nature remains neutral with regard to jurisdictional claims in published maps and institutional affiliations.

\section{Author details}

${ }^{1}$ Vinmec Research Institute of Stem Cell and Gene Technology, 458 Minh Khai, Hai Ba Trung, Hanoi, Vietnam. ${ }^{2}$ Department of Information Technology, Ghent University - imec, Ghent, Belgium. ${ }^{3} \mathrm{VNU}$ University of Science, Vietnam National University, Hanoi, Vietnam. ${ }^{4}$ Faculty of Biology, Hanoi National University of Education, Hanoi, Vietnam. ${ }^{5}$ Institute of Research and Development, Duy Tan University, 03 Quang Trung, Da Nang, Vietnam.

${ }^{6}$ Faculty of Information Technology, Ton Duc Thang University, Ho Chi Minh City, Vietnam.

Received: 13 March 2017 Accepted: 6 November 2017

Published online: 14 November 2017

Reference

1. He L, Hannon GJ. MicroRNAs: small RNAs with a big role in gene regulation. Nat Rev Genet. 2004;5(7):522-31.

2. Chen K, Rajewsky N. The evolution of gene regulation by transcription factors and microRNAs. Nat Rev Genet. 2007:8(2):93-103.

3. Conrad R, Barrier M, Ford LP. Role of miRNA and miRNA processing factors in development and disease. Birth Defects Res C Embryo Today. 2006;78(2):107-17.

4. Li Y, Kowdley KV. MicroRNAs in common human diseases. Genomics Proteomics Bioinformatics. 2012;10(5):246-53.

5. Mendell Joshua T, Olson Eric N. MicroRNAs in stress Signaling and human disease. Cell. 2012;148(6):1172-87.

6. Esteller M. Non-coding RNAs in human disease. Nat Rev Genet. 2011; 12(12):861-74.

7. Steinfeld I, Navon R, Ach R, Yakhini Z. miRNA target enrichment analysis reveals directly active miRNAs in health and disease. Nucleic Acids Res. 2013;41(3):e45.

8. Salvatore M, Magrelli A, Taruscio D. The role of microRNAs in the biology of rare diseases. Int J Mol Sci. 2011;12(10):6733-42.

9. Zeng X, Zhang X, Zou Q. Integrative approaches for predicting microRNA function and prioritizing disease-related microRNA using biological interaction networks. Brief Bioinform. 2016;17(2):193-203.

10. Jiang $Q$, Hao $Y$, Wang $G$, Juan $L$, Zhang $T$, Teng $M$, Liu $Y$, Wang $Y$. Prioritization of disease microRNAs through a human phenomemicroRNAome network. BMC Syst Biol. 2010;4(Suppl 1):S2.

11. Qinghua J, Yangyang H, Guohua W, Tianjiao Z, Yadong W. Weighted Network-Based Inference of Human MicroRNA-Disease Associations. In: Frontier of Computer Science and Technology (FCST), 2010 Fifth 
International Conference on: 18-22 Aug. 2010;2010:431-435. http:// ieeexplore.ieee.org/document/5575915/.

12. Wang D, Wang J, Lu M, Song F, Cui Q. Inferring the human microRNA functional similarity and functional network based on microRNA-associated diseases. Bioinformatics. 2010;26(13):1644-50.

13. Xu J, Li C-X, Li Y-S, Lv J-Y, Ma Y, Shao T-T, Xu L-D, Wang Y-Y, Du L, Zhang $Y-P$, et al. MiRNA-miRNA synergistic network: construction via co-regulating functional modules and disease miRNA topological features. Nucleic Acids Res. 2011;39(3):825-36.

14. Le D-H, Marchal K. Integration of miRNA-miRNA networks improves the prediction of novel disease associated miRNAs. In: The First NAFOSTED Conference on Information and Computer Science; Hanoi. 2014;438-448. http://cds.cern.ch/record/1996698?!n=sk.

15. Chen X, Liu M-X, Yan G-Y. RWRMDA: predicting novel human microRNAdisease associations. Mol BioSyst. 2012;8(10):2792-8.

16. Le D-H. Network-based ranking methods for prediction of novel disease associated microRNAs. Comput Biol Chem. 2015:58:139-48.

17. Xuan P, Han K, Guo M, Guo Y, Li J, Ding J, Liu Y, Dai Q, Li J, Teng Z, et al. Prediction of microRNAs associated with human diseases based on weighted k most similar Neighbors. PLoS One. 2013;8(8):e70204.

18. Chen $\mathrm{H}$, Zhang $Z$. Similarity-based methods for potential human microRNAdisease association prediction. BMC Med Genet. 2013;6(1):12.

19. Chen $H$, Zhang Z. Prediction of associations between OMIM diseases and MicroRNAs by random walk on OMIM disease similarity network. Sci World J. 2013;2013:6.

20. Le D-H: Disease phenotype similarity improves the prediction of novel disease-associated microRNAs. In: Information and Computer Science (NICS), 2015 2nd National Foundation for Science and Technology Development Conference on: 16-18 Sept. 2015;2015:76-81. http://ieeexplore.ieee.org/ document/7302226/.

21. You Z-H, Huang Z-A, Zhu Z, Yan G-Y, Li Z-W, Wen Z, Chen X. PBMDA: a novel and effective path-based computational model for miRNA-disease association prediction. PLoS Comput Biol. 2017;13(3):e1005455.

22. Chen $X$, Yan CC, Zhang X, You Z-H, Huang Y-A, Yan G-Y. HGIMDA heterogeneous graph inference for miRNA-disease association prediction. Oncotarget. 2016;7(40):65257-69.

23. Chen X, Yan CC, Zhang X, You Z-H, Deng L, Liu Y, Zhang Y, Dai Q. WBSMDA: Within and Between Score for MiRNA-Disease Association prediction. Scientific Reports. 2016;6:21106.

24. Chen X, Wu Q-F, Yan G-Y. RKNNMDA: ranking-based KNN for MiRNA-disease association prediction. RNA Biol. 2017;14(7):952-62.

25. Chen X, Jiang Z-C, Xie D, Huang D-S, Zhao Q, Yan G-Y, You Z-H. A novel computational model based on super-disease and miRNA for potential miRNA-disease association prediction. Mol BioSyst. 2017; 13(6):1202-12.

26. Bartel DP. MicroRNAs: genomics, biogenesis, mechanism, and function. Cell. 2004:116(2):281-97.

27. Lu M, Zhang Q, Deng M, Miao J, Guo Y, Gao W, Cui Q. An analysis of human MicroRNA and disease associations. PLoS One. 2008;3(10):e3420.

28. Shi H, Xu J, Zhang G, Xu L, Li C, Wang L, Zhao Z, Jiang W, Guo Z, Li $X$. Walking the interactome to identify human miRNA-disease associations through the functional link between miRNA targets and disease genes. BMC Syst Biol. 2013;7(1):1-12.

29. Jiang $Q$, Wang $G$, Wang $Y$ : An approach for prioritizing disease-related microRNAs based on genomic data integration. In: Biomedical Engineering and Informatics (BMEI), 2010 3rd International Conference on: 2010. IEEE: 2270-2274. http://ieeexplore.ieee.org/document/ $5639313 /$.

30. Qinghua J, Guohua W, Tianjiao Z, Yadong W: Predicting human microRNAdisease associations based on support vector machine. In: Bioinformatics and Biomedicine (BIBM), 2010 IEEE International Conference on: 18-21 Dec. 2010;2010:467-472. http://ieeexplore.ieee.org/document/5706611/.

31. Qabaja A, Alshalalfa M, Bismar T, Alhajj R. Protein network-based lasso regression model for the construction of disease-miRNA functional interactions. EURASIP J Bioinforma Syst Biol. 2013;2013(1):3

32. Xu J, Li C-X, Lv J-Y, Li Y-S, Xiao Y, Shao T-T, Huo X, Li X, Zou Y, Han Q-L, et al. Prioritizing candidate disease miRNAs by topological features in the miRNA target-Dysregulated network: case study of prostate cancer. Mol Cancer Ther. 2011;10(10):1857-66.

33. Chen $X$, Yan G-Y. Semi-supervised learning for potential human microRNAdisease associations inference. Sci Rep. 2014;4:5501.
34. Bentwich I, Avniel A, Karov Y, Aharonov R, Gilad S, Barad O, Barzilai A, Einat $P$, Einav $U$, Meiri E. Identification of hundreds of conserved and nonconserved human microRNAs. Nat Genet. 2005;37(7):766-70.

35. Kozomara A. Griffiths-Jones S: miRBase: annotating high confidence microRNAs using deep sequencing data. Nucleic Acids Res. 2014; 42(D1):D68-73.

36. Friedman RC, Farh KK-H, Burge CB, Bartel DP. Most mammalian mRNAs are conserved targets of microRNAs. Genome Res. 2009;19(1):92-105.

37. Lewis BP, Burge CB, Bartel DP. Conserved seed pairing, often flanked by adenosines, indicates that thousands of human genes are microRNA targets. Cell. 2005;120(1):15-20.

38. Tan Gana NH, Victoriano AFB, Okamoto T. Evaluation of online miRNA resources for biomedical applications. Genes Cells. 2012;17(1):11-27.

39. Satoh J-i, Tabunoki H. Comprehensive analysis of human microRNA target networks. BioData Mining. 2011:4(1):17.

40. Vaishnavi V, Manikandan M, Tiwary BK, Munirajan AK. Insights on the functional impact of MicroRNAs present in autism-associated copy number variants. PLoS One. 2013;8(2):e56781.

41. Pasquinelli AE. MicroRNAs and their targets: recognition, regulation and an emerging reciprocal relationship. Nat Rev Genet. 2012;13(4):271-82.

42. Li Y, Qiu C, Tu J, Geng B, Yang J, Jiang T, Cui Q. HMDD v2.0: a database for experimentally supported human microRNA and disease associations. Nucleic Acids Res. 2014;42(D1):D1070-4.

43. Dweep H, Sticht C, Pandey P, Gretz N. miRWalk - database: prediction of possible miRNA binding sites by "walking" the genes of three genomes. J Biomed Inform. 2011;44(5):839-47.

44. Lewis BP, Shih I, Jones-Rhoades MW, Bartel DP, Burge CB. Prediction of mammalian MicroRNA targets. Cell. 2003;115(7):787-98.

45. Jiang $Q$, Wang $Y$, Hao $Y$, Juan L, Teng M, Zhang X, Li M, Wang G, Liu Y. miR2Disease: a manually curated database for microRNA deregulation in human disease. Nucleic Acids Res. 2009;37(suppl 1):D98-D104.

46. van Driel MA, Bruggeman J, Vriend G, Brunner HG, Leunissen JAM. A textmining analysis of the human phenome. Eur J Hum Genet. 2006;14(5):535-42.

47. Amberger J, Bocchini CA, Scott AF, Hamosh A. McKusick's online Mendelian inheritance in man (OMIM $\left.{ }^{\circledast}\right)$. Nucleic Acids Res. 2009;37(suppl 1):D793-6.

48. Chen X, You Z-H, Yan G-Y, Gong D-W. IRWRLDA: improved random walk with restart for IncRNA-disease association prediction. Oncotarget. 2016; 7(36):57919.

49. Le D-H, Kwon Y-K. Neighbor-favoring weight reinforcement to improve random walk-based disease gene prioritization. Comput Biol Chem. 2013;44(0):1-8.

50. Chen X, Liu M-X, Yan G-Y. Drug-target interaction prediction by random walk on the heterogeneous network. Mol BioSyst. 2012;8(7):1970-8.

51. Chen X. miREFRWR: a novel disease-related microRNA-environmental factor interactions prediction method. Mol BioSyst. 2016;12(2):624-33.

52. Feldman I, Rzhetsky A, Vitkup D. Network properties of genes harboring inherited disease mutations. Proc Natl Acad Sci. 2008:105(11):4323-8.

53. Goh K-I, Cusick ME, Valle D, Childs B, Vidal M, Barabási A-L. The human disease network. Proc Natl Acad Sci. 2007:104(21):8685-90.

54. Oti M, Brunner HG. The modular nature of genetic diseases. Clin Genet. 2007;71(1):1-11.

55. Maglott D, Ostell J, Pruitt KD, Tatusova T. Entrez gene: gene-centered information at NCBI. Nucleic Acids Res. 2011;39(suppl 1):D52-7.

56. Rice J. Mathematical statistics and data analysis: Cengage learning; 2006 https://books.google.com.vn/books/about/Mathematical_Statistics_and_ Data_Analysi.html?id=EKAyeX2GVgC\&redir_esc=y.

57. Kozlowska E, Krzyzosiak W, Koscianska E. Regulation of Huntingtin gene expression by miRNA-137, -214, -148a, and their respective isomiRs. Int J Mol Sci. 2013;14(8):16999.

58. Shi C, Huang F, Gu X, Zhang M, Wen J, Wang X, You L, Cui X, Ji C, Guo X. Adipogenic miRNA and meta-signature miRNAs involved in human adipocyte differentiation and obesity. Oncotarget. 2016;7(26):40830.

59. Nicholas LM, Rattanatray L, MacLaughlin SM, Ozanne SE, Kleemann DO, Walker SK, Morrison JL, Zhang S, Muhlhäusler BS, Martin-Gronert MS, et al. Differential effects of maternal obesity and weight loss in the periconceptional period on the epigenetic regulation of hepatic insulinsignaling pathways in the offspring. FASEB J. 2013;27(9):3786-96.

60. Marques FZ, Campain AE, Tomaszewski M, Zukowska-Szczechowska E, Yang YHJ, Charchar FJ, Morris BJ. Gene expression profiling reveals Renin mRNA Overexpression in human hypertensive kidneys and a role for MicroRNAs. Hypertension. 2011;58(6):1093-8. 
61. Wu S, Gu Y, Huang Y, Wong T-C, Ding H, Liu T, Zhang Y, Zhang X. Novel biomarkers for non-functioning invasive pituitary adenomas were identified by using analysis of microRNAs expression profile. Biochem Genet. 2017; 55(3):253-67.

62. Zhou B, Chu M, Xu S, Chen X, Liu Y, Wang Z, Zhang F, Han S, Yin J, Peng B, et al. Hsa-let-7c-5p augments enterovirus 71 replication through viral subversion of cell signaling in rhabdomyosarcoma cells. Cell Bioscience. 2017;7(1):7.

63. Meola N, Gennarino V, Banfi S. microRNAs and genetic diseases. Patho Genetics. 2009;2(1):7.

Submit your next manuscript to BioMed Central and we will help you at every step:

- We accept pre-submission inquiries

- Our selector tool helps you to find the most relevant journal

- We provide round the clock customer support

- Convenient online submission

- Thorough peer review

- Inclusion in PubMed and all major indexing services

- Maximum visibility for your research

Submit your manuscript at www.biomedcentral.com/submit 